\title{
Coronary artery bypass surgery
}

\section{compared with percutaneous coronary} interventions in patients with insulin-treated type 2 diabetes mellitus: a systematic review and meta-analysis of 6 randomized controlled trials

\author{
Pravesh Kumar Bundhun, Zi Jia Wu and Meng-Hua Chen*
}

\begin{abstract}
Background: Data regarding the long-term clinical outcomes in patients with insulin-treated type 2 diabetes mellitus (ITDM) revascularized by either coronary artery bypass surgery (CABG) or percutaneous coronary intervention (PCI) are still controversial. We sought to compare the long-term ( $\geq 1$ year) adverse clinical outcomes in patients with ITDM who underwent revascularization by either $\mathrm{CABG}$ or $\mathrm{PCl}$.

Methods: Randomized Controlled Trials (RCTs) comparing the long-term clinical outcomes in patients with ITDM and non-ITDM revascularized by either CABG or PCI were searched from electronic databases. Data for patients with ITDM were carefully retrieved. Odd Ratio (OR) with $95 \%$ confidence interval (CI) was used to express the pooled effect on discontinuous variables and the pooled analyses were performed with RevMan 5.3.

Results: Six RCTs involving 10 studies, with a total of 1297 patients with ITDM were analyzed (639 patients from the $C A B G$ group and 658 patients from the $\mathrm{PCl}$ group). CABG was associated with a significantly lower mortality rate compared to PCI with OR: $0.59,95 \% \mathrm{Cl} 0.42-0.85 ; \mathrm{P}=0.004$. Major adverse cardiovascular and cerebrovascular events as well as repeated revascularization were also significantly lower in the CABG group with OR: $0.51,95 \%$ Cl 0.27-0.99; $\mathrm{P}=0.03$ and $\mathrm{OR} 0.34,95 \% \mathrm{Cl} 0.24-0.49 ; \mathrm{P}<0.00001$ respectively. However, compared to $\mathrm{PCl}$, the rate of stroke was higher in the CABG group with OR: $1.41,95 \% \mathrm{Cl} 0.64-3.09 ; \mathrm{P}=0.40$, but this result was not statistically significant.
\end{abstract}

Conclusion: $C A B G$ was associated with significantly lower long-term adverse clinical outcomes compared to $\mathrm{PCl}$ in patients with ITDM. However, due to an insignificantly higher rate of stroke in the CABG group, further researches with a larger number of randomized patients are required to completely solve this issue.

Keywords: Percutaneous coronary intervention, Coronary artery bypass surgery, Insulin-treated diabetes mellitus, Adverse clinical outcomes

\section{Background}

Type 2 Diabetes mellitus (T2DM) and Coronary Artery Disease (CAD) are two important and prevalent chronic

\footnotetext{
*Correspondence: xyicucmh@sina.com

Institute of Cardiovascular Diseases, the First Affiliated Hospital of Guangxi

Medical University, Nanning, Guangxi 530027, Peoples' Republic of China
}

disorders which often co-exist [1]. Patients with T2DM have been found to have more adverse clinical outcomes compared to non-diabetic (NDM) patients after Percutaneous Coronary Intervention (PCI). Moreover, prognosis after coronary angioplasty is even worse in insulin-treated T2DM (ITDM) patients when compared to non-insulin treated T2DM (NITDM) patients [2,3]. 
Many studies have shown that revascularization performed using Coronary Artery Bypass Surgery (CABG) appears to be a better option compared to $\mathrm{PCI}$ in patients with T2DM, particularly in conditions such as multi-vessel CAD, chronic total occlusion and so on. For example, earlier reports based on data from the CARDia (Coronary Artery Revascularization in Diabetes) trial [4] and the 5-year results of the SYNTAX trial [5] indicated significantly higher rates of Major Adverse Cardiovascular and Cerebrovascular Events (MACCEs) associated with PCI compared to CABG in patients with T2DM.

However, even if $\mathrm{PCI}$ is expected to be associated with worse clinical outcomes in patients with ITDM, data regarding the long-term adverse clinical outcomes in similar patients revascularized by either CABG or PCI are still controversial. To further support this point, results from the FREEDOM trial showed no significant difference in the magnitude of CABG versus PCI treatment effect in patients with ITDM [2].

Therefore, to solve this issue, we aim to compare the long-term adverse clinical outcomes in patients with ITDM revascularized by either CABG or PCI.

\section{Methods}

Data sources and search strategy

Medline and EMBASE were searched for Randomized Controlled Trials (RCTs) comparing CABG with PCI in patients with both ITDM and NITDM by typing the words 'diabetes mellitus, coronary artery bypass surgery and percutaneous coronary intervention. To further enhance this search, the abbreviations 'DM, CABG and PCI' have also been used. References have also been checked for relevant RCTs. No language restriction was applied.

\section{Inclusion and exclusion criteria}

Studies were included if:

(a) They were RCTs.

(b) They compared patients with ITDM and NITDM revascularized by either CABG or PCI.

(c) They reported long-term ( $\geq 1$ year) adverse clinical outcomes observed in those patients with ITDM.

Studies were excluded if:

(a) They were not RCTs (excluded if they were observational studies, case studies or meta-analyses).

(b) Data for patients with ITDM could not be retrieved from the studies.

(c) They did not compare CABG with PCI in patients with ITDM. (d) They had a short-term ( $<1$ year) follow up period.

\section{Types of participants}

All the patients suffered from T2DM and were treated with insulin therapy. The patients were either randomized to undergo revascularization by CABG or PCI.

\section{Outcomes and definitions}

- Adverse clinical outcomes such as all-cause mortality, stroke, MACCEs, Myocardial infarction (MI) and repeated or further revascularization during a longterm follow-up period ( $\geq 1$ year) were considered as the clinical endpoints in this study. Reported clinical outcomes and follow up periods have been represented in Table 1.

- MACCEs included all-cause death, cerebrovascular accident (CVA), MI or repeat revascularization (subsequent to PCI or CABG).

- Stroke was defined as focal neurological deficits of central origin lasting $>72 \mathrm{~h}$, resulting in permanent brain damage or body impairment.

- Death was defined as all-cause death. If data for all cause death was not available, data for cardiac death have been used.

\section{Data extraction and quality assessment}

Data were reviewed and assessed for eligibility and methodological quality by two authors (PKB and ZW). Information regarding study and patients with ITDM, intervention strategies, and the pre-specified clinical outcomes reported and the corresponding follow-up periods was systematically extracted. Disagreements were discussed between the authors, and if the authors could not reach a consensus, disagreements were resolved by the third author (M.H.C).

The bias risk of trials was assessed with the components recommended by the Cochrane Collaboration, including the following criteria: sequence generation of the allocation, allocation concealment, blinding of participants, personnel, and outcome assessors, incomplete outcome data, selective outcome reporting, and other sources of bias [6]. Trials have been carefully assessed and a score ranging from 0 to 12 points has been allocated to specific trials depending on whether they satisfied all the components recommended by the Cochrane Collaboration. Low risk of bias corresponded to a score of 2 in each of these 6 components whereas a score of 0 was given if this evaluation showed a high risk of bias in these RCTs. A score of 1 was reserved for unclear bias. Therefore, if a trial showed 'low risk bias' in all the 6 components recommended by the Cochrane Collaboration, a total score of $2 \times 6=12$ would be allocated to it. 
Table 1 Reported outcomes and follow up periods

\begin{tabular}{lll}
\hline Studies & Follow-up (years) & Clinical outcomes \\
\hline Banning [8] & 1 & Death, MI, stent thrombosis, repeated revascularization, MACCES \\
Bari [9] & 5 & Death \\
Dangas [2] & 1,5 & Death, stroke, MI, repeated revascularization, MACCEs \\
Detre [10] & 5 & Death \\
Farkouh [11] & $1,2,5$ & Death, MACCEs, MI, stroke, repeated revascularization \\
Kamalesh [12] & 1,2 & Death, stroke, MI, repeated revascularization \\
Kappetein [13] & 5 & Death, stroke, MI, repeated revascularization, stent thrombosis \\
Kapur [4] & 1 & Death, non-fatal MI, stroke, further revascularization, MACCEs \\
Lima [14] & 10 & Death \\
Soares [15] & $1,2-5$ & Death \\
\hline
\end{tabular}

MI myocardial infarction, MACCEs major adverse cerebrovascular and cardiovascular events

\section{Statistical analysis}

The assessment of heterogeneity across the studies was performed using the (a) Cochrane Q-statistic whereby a 'p value' less than 0.05 was considered statistically significant and, (b) Cochrane $\mathrm{I}^{2}$-statistic which represented the percentage of the total variation across studies that is due to heterogeneity rather than chance whereby an $\mathrm{I}^{2}$ value of $0 \%$ indicated no heterogeneity, and an increased heterogeneity was indicated by a larger value. If $\mathrm{I}^{2}$ was $<50 \%$, fixed effect was used. However, if $\mathrm{I}^{2}$ was $>50 \%$, a random effect has been used. Funnel plots were assessed for publication bias. We calculated odd ratios (OR) and $95 \%$ confidence intervals (CIs) for categorical variables. The pooled analyses were performed with RevMan 5.3 software.

\section{Ethics}

Ethical approval was not necessary as this study is a Systematic Review and Meta-Analysis.

\section{Results}

\section{Study selection and general features of the included} studies

Study selection, data collection, analysis, and reporting of the results were performed using the recommendations of the PRISMA (Preferred Reporting Items for Systematic Reviews and Meta-Analyses) statement [7].

Six Trials (involving 10 studies) have been included in this meta-analysis (Table 2). During the selection process, 20 studies comparing CABG with PCI in patients with T2DM were found. However, because data for patients with ITDM could not be retrieved from 10 studies, these studies had been excluded from this analysis. The flow diagram for the study selection has been represented in Fig. 1.

SYNTAX Trial, BARI Trial, FREEDOM Trial, CARDIa and MASS II Trials were included in this meta-analysis.
These 10 studies [2, 4, 8-15] reported long-term adverse clinical outcomes as their endpoints.

Patient enrollment occurred from the year 1988 to 2010. Randomization of the patients was performed in different medical centers mostly from New York, England and Brazil.

A total number of 1,297 patients with ITDM consisting of 639 patients from the CABG group and 658 patients from the PCI group were included in this meta-analysis. All patients provided signed consents. General features of these included trials have been listed in Table 2 .

Six studies reported a follow-up period of 1 year, two studies reported a follow-up period of 2 years, five studies reported a follow-up period of 5 years, one study reported a follow-up between 2 and 5 years and another study with a follow up period of 10 years (Table 1 ). To avoid repetition, trials were considered.

\section{Baseline characteristics}

Table 3 shows the baseline characteristics of the included studies. A mean age of about 60 years was observed in patients from both groups. With the exception of two studies, similar percentages of males were reported in the CABG and PCI groups. The percentages of hypertensive patients and smokers were also similar in both groups. Overall, there were no significant differences in the baseline features between these patients with ITDM classified in the CABG or PCI group.

The bias risk scores were as follow: Seven studies were allocated a score of 8 , two studies were allocated a score of 9 and one study was allocated a score of 10 . These scores have been listed in Table 3.

\section{Results of this meta-analysis}

The pooled analysis of these 1207 patients with ITDM showed CABG to be associated with a significantly lower long-term mortality rate with OR: 0.59, 95 \% CI 
Table 2 General features of the included studies

\begin{tabular}{|c|c|c|c|c|c|c|}
\hline Studies & Trial name & Study type & Region & $\begin{array}{l}\text { Randomization } \\
\text { period (year) }\end{array}$ & $\begin{array}{l}\text { Patients in CABG } \\
\text { group (n) }\end{array}$ & $\begin{array}{l}\text { Patients in } \mathrm{PCl} \\
\text { group }(\mathrm{n})\end{array}$ \\
\hline Banning (2010) & SYNTAX & $\mathrm{RCT}$ & England & - & 88 & 88 \\
\hline Bari (1997) & BARI & $\mathrm{RCT}$ & Pittsburgh & 1988-1991 & 47 & 45 \\
\hline Dangas (2014) & FREEDOM & $\mathrm{RCT}$ & New York & $2005-2010$ & 277 & 325 \\
\hline Detre (1999) & BARI & $\mathrm{RCT}$ & Pittsburgh & 1988-1991 & 80 & 78 \\
\hline Kamalesh (2013) & - & $\mathrm{RCT}$ & Indiana & $2006-2010$ & 45 & 48 \\
\hline Kappetein (2013) & SYNTAX & $\mathrm{RCT}$ & Netherlands & - & 93 & 89 \\
\hline Kapur (2010) & CARDla & $\mathrm{RCT}$ & England & - & 99 & 88 \\
\hline Lima (2013) & MASS ॥ & $\mathrm{RCT}$ & Brazil & 1995-2000 & 29 & 30 \\
\hline Soares (2006) & MASS ॥ & $\mathrm{RCT}$ & Brazil & 1995-2000 & 23 & 19 \\
\hline
\end{tabular}

$C A B G$ coronary artery bypass surgery, $P C l$ percutaneous coronary intervention, $R C T$ randomized controlled trials

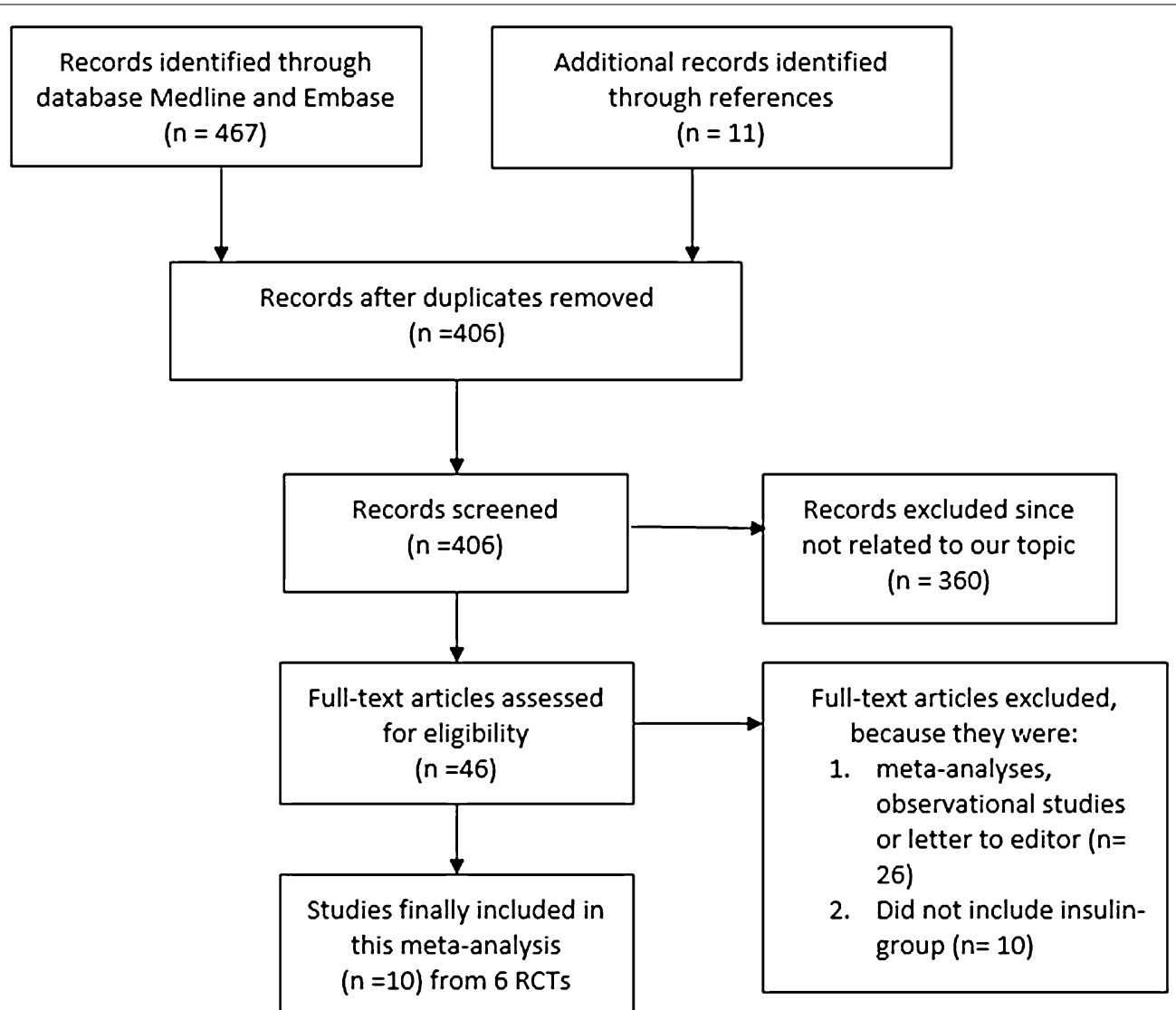

Fig. 1 Flow diagram of the study selection. 456 articles were identified from Medline and EMBASE and further 11 relevant articles were identified through reference lists of highly selective studies. After filtering the duplicates, 360 articles were excluded since they were not related to our topic. 46 full-text articles were assessed for eligibility. Meta-analyses, observational studies, and letters to editor were further eliminated ( $\mathrm{n}=26$ ). Studies including data for patients with ITDM which were unable to be retrieved were also eliminated $(n=10)$. Finally 6 RCTs involving 10 studies were selected for this systematic review and meta-analysis

0.42-0.85; $\mathrm{P}=0.004$. MACCEs and revascularization were also significantly lower in the CABG group with OR: 0.51, 95 \% CI 0.27-0.99; $\mathrm{P}=0.03$ and OR: 0.34,
$95 \%$ CI 0.24-0.49; $\mathrm{P}<0.00001$ respectively. MI insignificantly favored CABG with OR: $0.75,95 \%$ CI 0.46-1.20; $\mathrm{P}=0.23$. The rate of stroke was higher in the CABG 
Table 3 Baseline characteristics of the included studies

\begin{tabular}{llllll}
\hline Trials & $\begin{array}{l}\text { Age } \\
\text { (years) }\end{array}$ & Males (\%) & HT (\%) & Cs (\%) & Cochrane \\
& CABG/PCl & CABG/PCI & CABG/PCI & CABG/PCI & Bias score \\
\hline Banning [8] & $65.4 / 65.4$ & $71.0 / 71.0$ & $69.9 / 69.9$ & $15.8 / 15.8$ & 8 \\
Bari (1997) & $62.5 / 62.1$ & $58.0 / 56.0$ & $66.0 / 65.0$ & - & 9 \\
Dangas [2] & $62.6 / 62.6$ & $61.3 / 6.3$ & $87.5 / 87.5$ & $17.9 / 17.9$ & 8 \\
Detre [10] & $62.3 / 62.3$ & $57.0 / 57.0$ & $65.0 / 65.0$ & $65.0 / 65.0$ & 9 \\
Farkouh [11] & $63.1 / 63.2$ & $69.5 / 73.2$ & - & $16.6 / 14.8$ & 8 \\
Kamalesh & $62.1 / 62.7$ & $99.0 / 99.0$ & $95.7 / 96.0$ & $20.6 / 27.7$ & 8 \\
[12] & & & & & \\
Kappetein & $65.4 / 65.4$ & $71.0 / 71.0$ & $70.0 / 70.0$ & $16.0 / 16.0$ & 8 \\
[13] & & & & & \\
Kapur [4] & $63.6 / 64.3$ & $77.9 / 70.7$ & $76.6 / 76.6$ & $24.6 / 24.6$ & 10 \\
Lima [14] & $59.0 / 61.0$ & $72.0 / 56.0$ & $71.0 / 72.0$ & $34.0 / 17.0$ & 8 \\
Soares [15] & $60.0 / 61.0$ & $67.0 / 54.0$ & $73.0 / 73.0$ & - & 8 \\
\hline
\end{tabular}

$H T$ hypertension, Cs current smoker

group with OR: 1.41, 95 \% CI 0.64-3.09; P = 0.40. However, the result was not statistically significant. Detailed result of this meta-analysis has been tabulated (Table 4). Results for the adverse clinical outcomes between CABG and PCI, using a fixed effect model $\left(\mathrm{I}^{2}<50 \%\right)$ have been illustrated in Fig. 2. The result for MACCEs using a random effect model $\left(\mathrm{I}^{2}>50 \%\right)$ has been illustrated in Fig. 3.

At 1 year, mortality rate was similar in both the CABG and the PCI groups. However, during a follow up period of 5 years, mortality was significantly higher in the PCI group with OR: $0.56,95 \%$ CI $0.40-0.79 ; \mathrm{P}=0.001$. This result has been illustrated in Fig. 4.

For all of the above analyses, sensitivity analysis yielded consistent results. Based on a visual inspection of the funnel plot, there has been no evidence of publication bias for the included studies that assessed all clinical endpoints. The funnel plot has been represented in Fig. 5.

\section{Discussion}

\section{Aim of this study}

Several studies have shown insulin therapy to be associated with worse cardiovascular outcomes in patients with T2DM after revascularization with PCI [3]. However, data regarding the long-term adverse clinical outcomes in patients with ITDM revascularized by either CABG or PCI are still controversial and therefore, we aim to solve this issue in this meta-analysis.

\section{Results of this meta-analysis}

Our results showed long-term mortality rate $(9.23 \%$ versus $14.0 \%$ ), MACCEs (15.9 versus $23.4 \%$ ) and repeated revascularization (7.99 versus $19.4 \%$ ) to be significantly lower in the CABG group compared to the PCI group. Mortality during a follow-up period of 5 years was also
Table 4 Result of this meta-analysis

\begin{tabular}{llllr}
\hline $\begin{array}{l}\text { Long-term } \\
\text { outcomes }\end{array}$ & $\begin{array}{l}\text { Trials } \\
\text { analyzed }\end{array}$ & OR, 95 \% Cl & P value & $\mathbf{I}^{\mathbf{2} \%}$ \\
\hline Mortality & 6 & $0.59[0.42,0.85]$ & 0.004 & 4 \\
MI & 4 & $0.75[0.46,1.20]$ & 0.23 & 26 \\
MACCEs & 3 & $0.51[0.27,0.99]$ & 0.03 & 72 \\
Stroke & 4 & $1.41[0.64,3.09]$ & 0.40 & 0 \\
Revascularization & 5 & $0.34[0.24,0.49]$ & $<0.00001$ & 38 \\
Mortality at 1 year & 5 & $1.07[0.52,2.19]$ & 0.86 & 0 \\
Mortality during & 4 & $0.56[0.40,0.79]$ & 0.001 & 0 \\
$\quad 5$ years & & & & \\
\hline
\end{tabular}

MI myocardial infarction, MACCEs major adverse cerebrovascular and cardiovascular events, $\mathrm{OR}$ odd ratio, $\mathrm{Cl}$ confidence interval

significantly lower in the CABG group (12.5 versus $19.9 \%)$. However, stroke which was higher in the CABG group with a percentage of $2.83 \%$ compared to $2.01 \%$ in those patients from the PCI group, was not statistically significant in this analysis.

\section{Other researches supporting our study}

Several studies showed similar results with this current meta-analysis. The systematic review and Bayesian network meta-analysis comparing the long-term outcomes between the revascularization techniques of PCI and CABG in patients with T2DM also showed an increased association of cardiovascular outcomes in the PCI group and therefore, concluded that CABG seemed to be the preferred revascularization strategy in such patients especially if long-term survival was to be considered [16]. Another recent meta-analysis of several randomized controlled trials comparing CABG with PCI found significantly lower mortality rates among patients with T2DM revascularized by $C A B G$ compared to those patients revascularized by PCI [17]. Moreover, the meta-analysis by Smit et al. comparing CABG with PCI in patients with CAD showed that lower rates of death and repeated revascularization were associated with CABG especially in those patients with T2DM, but however, CABG was associated with a significantly higher risk of stroke in his study [18]. These meta-analyses recently discussed above, compared revascularization by CABG and PCI in patients with CAD or T2DM (consisting of patients with both ITDM and NITDM). Our study focused only on patients with ITDM.

\section{Is insulin therapy responsible for these adverse cardiovascular outcomes?}

Insulin therapy could be one of the reasons responsible for the increased adverse cardiovascular outcomes among patients who underwent revascularization procedures (including both CABG and PCI). Even if the study by Marso et al. showed no significant differences 


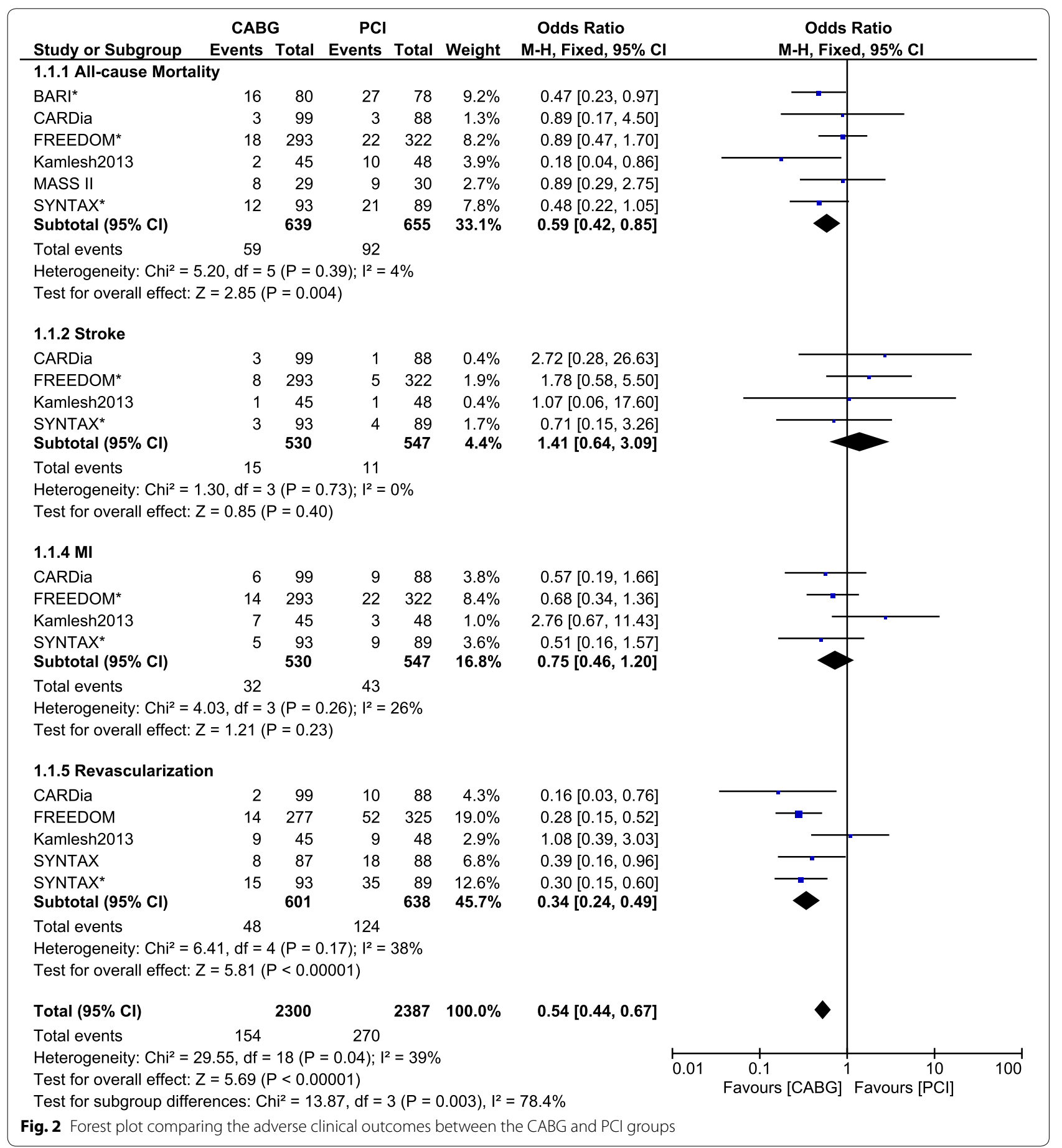

in treatment effect between CABG and PCI in patients with T2DM, the author concluded that insulin therapy remained an independent risk of adverse outcomes in patients with T2DM [19]. Moreover, the prospective registry data of consecutive CABG patients reported mortality to be significantly higher in patients with T2DM treated with insulin therapy, compared to those patients not treated with insulin, or NDM patients [20].

However, insulin therapy is expected to be only partly responsible for these adverse clinical events after revascularization. The review published by Lee et al. which showed a significantly lower rate of mortality and 


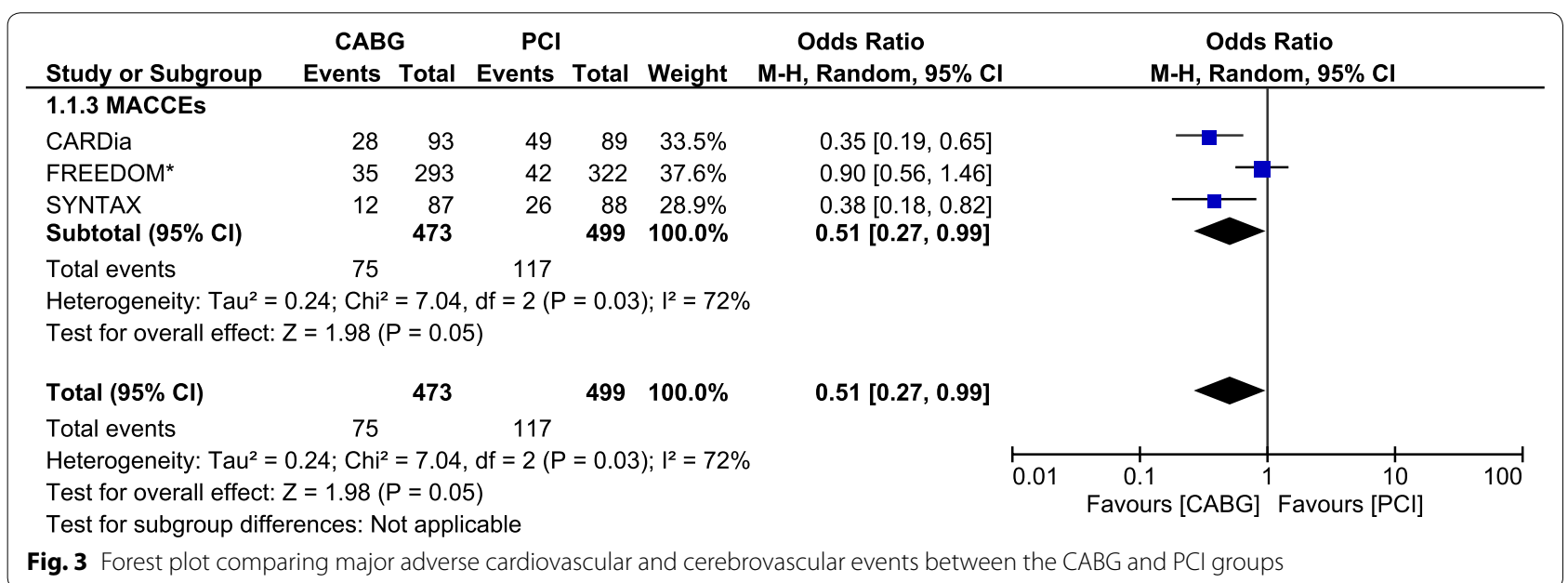

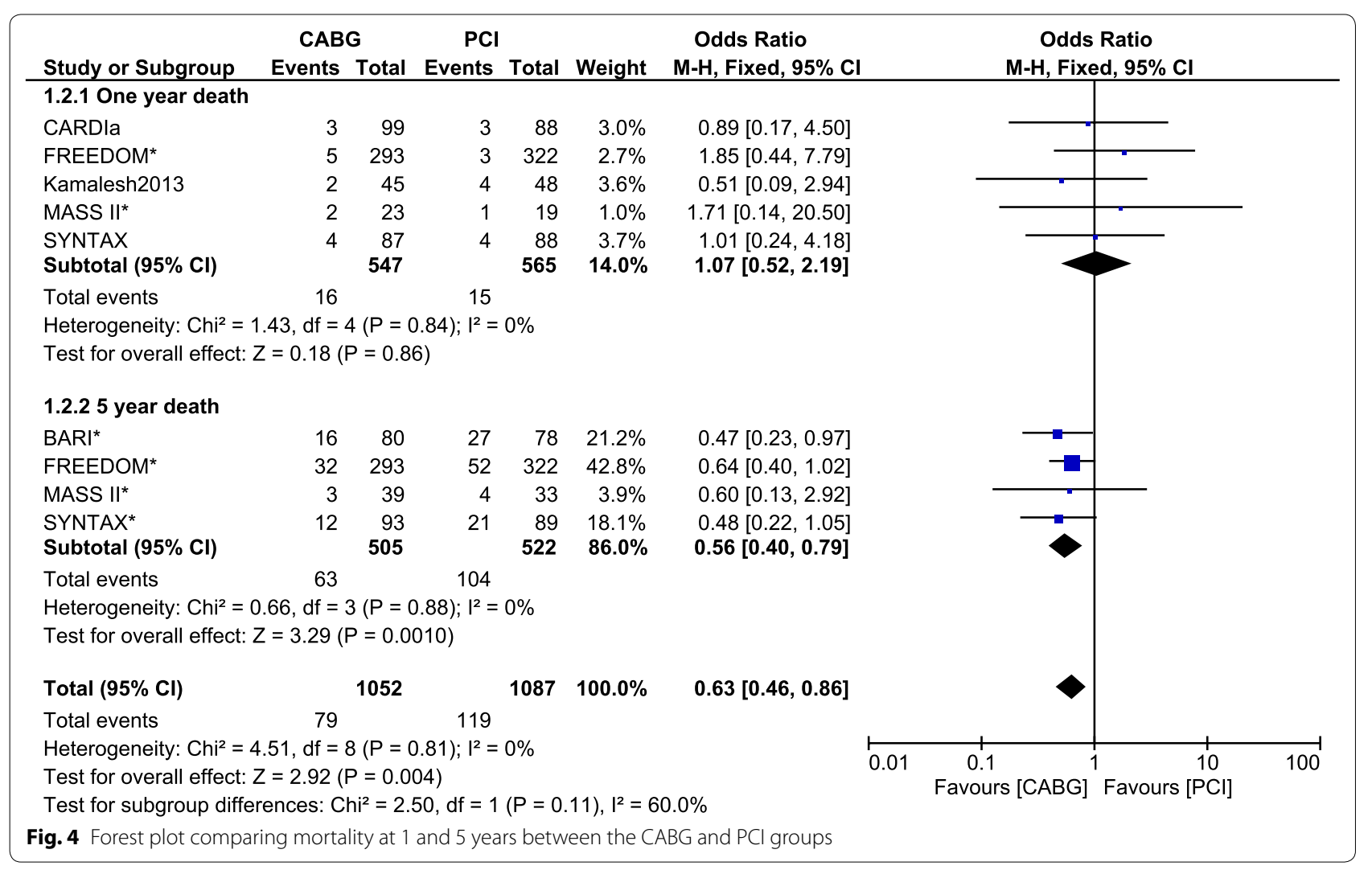

MACCEs among patients with T2DM in the CABG group, concluded that insulin therapy did not affect the clinical outcomes reported in these two revascularization procedures [21]. Also, the study by Liu et al. showed an increased level of HBA1c to be an independent predictor of MACCEs in similar patients [22]. Furthermore, the study by Lopez de Andres et al. showed a higher comorbidity and the female gender to be associated with a high rate of in-hospital mortality in patients with T2DM and
NDM [23]. Patients with T2DM often exhibit increased platelet reactivity despite combined treatment with clopidogrel and aspirin after PCI [24]. Patients with ITDM requiring prasugrel after $\mathrm{PCI}$ also have higher platelet reactivity compared to patients with NITDM or those without T2DM. This could also contribute to a higher risk of cardiovascular outcomes after revascularization procedures. However, our study was different since it compared CABG and PCI in patients with ITDM. 


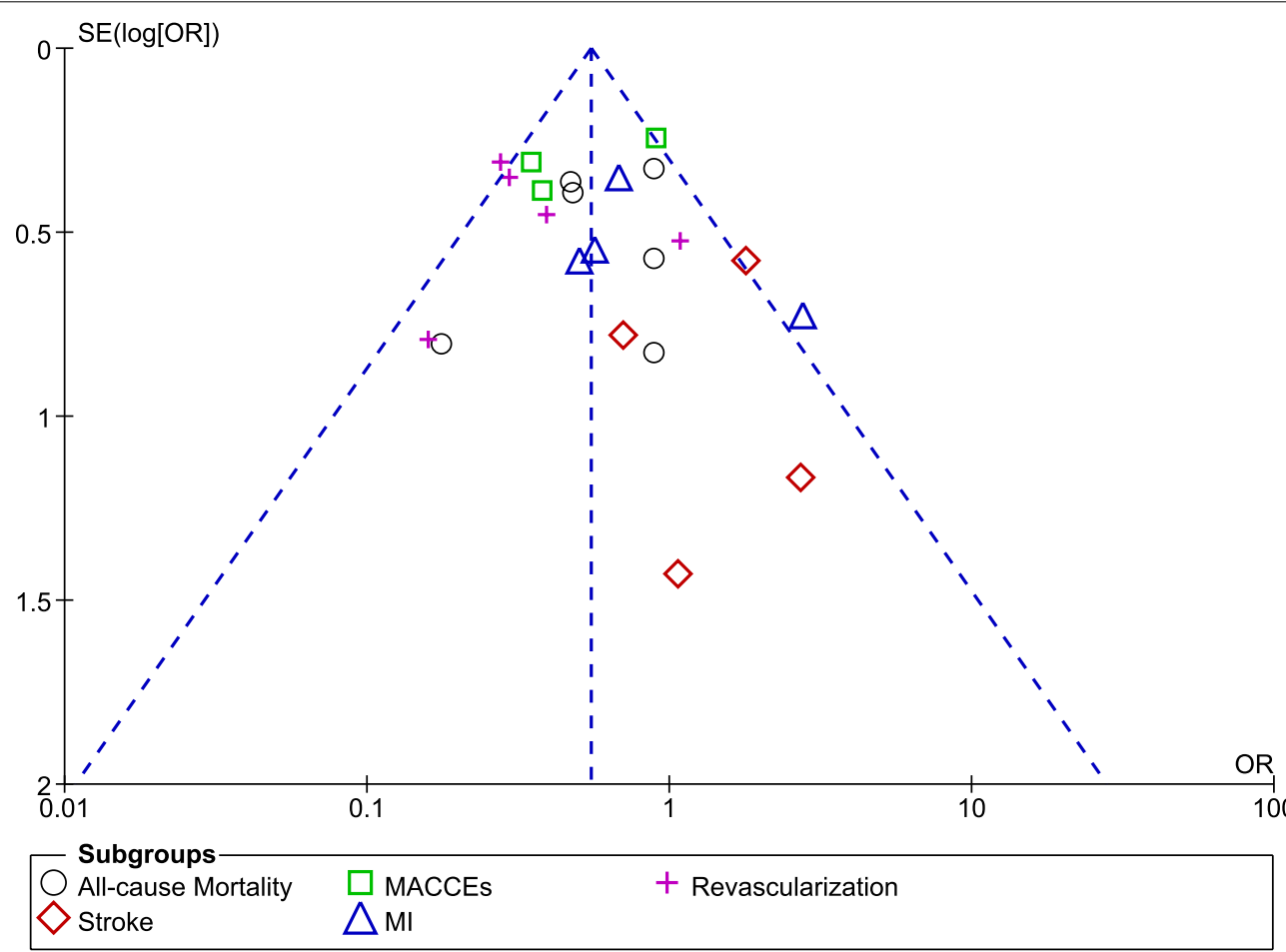

Fig. 5 Funnel plot assessing publication bias

\section{Other researches with different results from our meta-analysis}

Other studies showed different results compared to ours. For example, the study by Gargiolo et al. comparing the 5 years clinical outcomes between CABG and PCI showed no statistical difference in mortality, MI and stroke between CABG and PCI but showed repeated revascularization which was significantly increased in the PCI group [25]. However, his study only included patients with left main coronary artery disease. Another study, by Hallberg et al. which assessed the association of DM with 16 years survival after revascularization by CABG showed patients without DM to have a similar survival rate with the other patients, while the mortality rate in patients with T2DM started to increase a few years post-CABG [26]. Apart from showing that DM was associated with an increased risk of mortality after CABG, his study also showed an even higher cardiovascular cause of mortality in patients with ITDM compared to those without insulin therapy but however, the study did not compare the adverse cardiovascular outcomes between CABG and PCI. Moreover, Naito et al. compared the mortality rate between CABG and PCI in elderly patients with T2DM complicated with multi-vessel coronary disease and showed no significant difference in the mortality rate between CABG and PCI [27]. But the author stated that the CABG group had more patients with complex coronary lesions which could be responsible for such an outcome.

Even if the rate of stroke was not statistically significant in our study, a few other RCTs have reported a significant increased risk of stroke in those patients revascularized by CABG compared to PCI. However, all these studies were not powerful enough to examine strict differences in the risk of stroke. Two prior meta-analyses have analyzed the risk of stroke post-CABG or post-PCI, with conflicting results $[28,29]$. But, another meta-analysis including 19 trials with 10,944 patients randomized to CABG and PCI showed a real association of an increased risk of stroke at 30 days and at mid-term follow up in the CABG group compared to the PCI group [30]. PostCABG versus post-PCI stroke rates (post-CABG stroke rate at 1 year was $1.83 \%$; with OR 1.67). Nevertheless, the increased stroke rate following CABG compared to PCI was independent of ITDM status (reported as 5-year rates of 7.5 vs. $3.7 \%$ for CABG and PCI in patients with ITDM; and 4.3 vs. $1.7 \%$ for CABG and PCI in patients with NITDM) and could mean an association of stroke with CABG and not with the insulin therapy.

\section{Novelty in this study}

This study has strictly been conducted in patients with ITDM. Several studies have compared CABG with PCI in 
patients with CAD or T2DM (including a combination of patients with both ITDM and NITDM). However, since patients with ITDM are more complicated patients with several co-morbidities, we have conducted a meta-analysis on this particular population of patients with T2DM.

Finally, similar to the FREEDOM trial which clearly showed revascularization by CABG to be superior compared to PCI in patients with T2DM, our study which also showed CABG to be better than PCI in patients with ITDM, also suggests a comparative analysis of the new incoming stents which warrant further research [31].

Our study is expected to satisfy all the requirements for a meta-analysis, in terms of low heterogeneity in almost all of the different subgroups, absent publication bias, and sensitivity analysis, and provides robust scientific validity to our findings, which can assist informed decision making by patients and physicians when deciding on the optimal strategy for revascularization in CAD patients with ITDM.

\section{Limitation}

Due to the small population size, the result of this study could be restricted to an extent. Moreover, these RCTs included patients with ITDM with different clinical conditions or complications. For example, the CARDia and FREEDOM trials included patients with symptomatic multi-vessel CAD and multi-vessel CAD with or without symptoms respectively whereas the SYNTAX trial included patients with left main coronary disease and/ or three vessel disease. The BARI trial included patients with severe CAD. Variable degree of complications in these patients could have an effect on our results. However, despite these limitations, our data point to the urgent need for comprehensive comparison between these two revascularization strategies.

\section{Conclusion}

Compared to PCI, CABG was associated with a significantly lower mortality rate, MACCEs and repeated revascularization during this long-term follow up period in patients with ITDM. However, even if a higher rate of stroke in the CABG group was not statistically significant in our result, new researches with larger number of randomized patients are required to completely solve this issue.

\footnotetext{
Abbreviations

CABG: coronary artery bypass grafting; $\mathrm{PCl}$ : percutaneous coronary intervention; T2DM: type 2 diabetes mellitus; ITDM: insulin-treated diabetes mellitus; MACCEs: major adverse cardiovascular and cerebrovascular events.
}

\section{Authors' contributions}

PKB was responsible for the conception and design, acquisition of data, analysis and interpretation of data, drafting the initial manuscript and revising it critically for important intellectual content. WZJ was responsible for the conception and design, acquisition of data, analysis and interpretation of data, drafting the initial manuscript and revising it critically for important intellectual content. M-HC was responsible for the conception and design, acquisition of data, interpretation of data, and for revising the manuscript critically for important intellectual content. All authors read and approved the final manuscript.

\section{Authors' information}

Pravesh Kumar Bundhun is the first author.

\section{Acknowledgements}

No writing assistance was required and there was no external source of funding for this research.

\section{Competing interests}

The authors declare that they have no competing interests.

Received: 17 September 2015 Accepted: 28 December 2015

Published online: 06 January 2016

\section{References}

1. Hammound T, Tanguay JF, Bourassa MG. Management of coronary artery disease: therapeutic options in patients with diabetes. J Am Coll Cardiol. 2000;36:355-65.

2. Dangas GD, Farkouh ME, Sleeper LA, Yang M, Schoos MM, Macaya C, Abizaid A, Buller CE, Devlin G, Rodriguez AE, Lansky AJ, Siami FS, Domanski $M$, Fuster $V_{;}$FREEDOM Investigators. Long-term outcome of PCI versus CABG in insulin and non-insulin-treated diabetic patients: results from the FREEDOM trial. J Am Coll Cardiol. 2014;64(12):1189-97.

3. Bundhun PK, Li N, Chen MH. Adverse cardiovascular outcomes between insulin-treated and non-insulin treated diabetic patients after percutaneous coronary intervention: a systematic review and meta-analysis. Cardiovasc Diabetol. 2015;14(1):135.

4. Kapur A, Hall RJ, Malik IS, et al. Randomized comparison of percutaneous coronary intervention with coronary artery bypass grafting in diabetic patients. 1-year results of the CARDia (Coronary Artery Revascularization in Diabetes) trial. J Am Coll Cardiol. 2010;55:432-40.

5. Sianos G, Morel MA, Kappetein AP, et al. The SYNTAX score: an angiographic tool grading the complexity of coronary artery disease. Eurolntervention. 2005;1:219-27.

6. Higgins JPT, Altman DG. Assessing risk of bias in included studies. In: Higgins JPT, Green S, editors. Cochrane handbook for systematic reviews of interventions. Wiley. 2008. p. 187-241.

7. Liberati A, Altman DG, Tetzlaff J, Mulrow C, Gøtzsche PC, loannidis JP, et al. The PRISMA statement for reporting systematic reviews and meta-analyses of studies that evaluate healthcare interventions: explanation and elaboration. BMJ. 2009;339:b2700.

8. Banning AP, Westaby S, Morice MC, Kappetein AP, Mohr FW, Berti S, Glauber M, Kellett MA, Kramer RS, Leadley K, Dawkins KD, Serruys PW. Diabetic and nondiabetic patients with left main and/or 3-vessel coronary artery disease: comparison of outcomes with cardiac surgery and paclitaxeleluting stents. J Am Coll Cardiol. 2010;55(11):1067-75.

9. Influence of diabetes on 5-year mortality and morbidity in a randomized trial comparing CABG and PTCA inpatients with multivessel disease: the Bypass Angioplasty Revascularization Investigation (BARI). Circulation. 1997; 96(6):1761-9.

10. Detre KM, Guo P, Holubkov R, Califf RM, Sopko G, Bach R, Brooks MM, Bourassa MG, Shemin RJ, Rosen AD, Krone RJ, Frye RL, Feit F. Coronary revascularization in diabetic patients: a comparison of the randomized and observational componentsof the Aypass Angioplasty Revascularization Investigation (BARI). Circulation. 1999;99(5):633-40.

11. Farkouh ME, Domanski M, Sleeper LA, Siami FS, Dangas G, Mack M, Yang $M$, Cohen DJ, Rosenberg Y, Solomon SD, Desai AS, Gersh BJ, Magnuson EA, Lansky A, Boineau R, Weinberger J, Ramanathan K, Sousa JE, Rankin J, Bhargava B, Buse J, Hueb W, Smith CR, Muratov V, Bansilal S, King S 3rd, Bertrand M, Fuster V, FREEDOM Trial Investigators. Strategies for multivessel revascularization in patients with diabetes. N Engl J Med. 2012;367(25):2375-84. 
12. Kamalesh M, Sharp TG, Tang XC, Shunk K, Ward HB, Walsh J, King S 3rd, Colling C, Moritz T, Stroupe K, Reda D, VA CARDS Investigators. Percutaneous coronary intervention versus coronary bypass surgery in United States veterans with diabetes. J Am Coll Cardiol. 2013;61(8):808-16.

13. Kappetein AP, Head SJ, Morice M-C, et al. Treatment of complex coronary artery disease in patients with diabetes: 5-year results comparing outcomes of bypass surgery and percutaneous coronary intervention in the SYNTAX trial. Eur J Cardiothorac Surg. 2013;43:1006-13.

14. Lima EG, Hueb W, Garcia RM, Pereira AC, Soares PR, Favarato D, Garzillo CL, D'Oliveira Vieira R, Rezende PC, Takiuti M, Girardi P, Hueb AC, Ramires JA, Kalil Filho R. Impact of diabetes on 10-year outcomes of patients with multivessel coronary artery disease in the Medicine, Angioplasty, or Surgery Study II (MASS II) trial. Am Heart J. 2013;166(2):250-7.

15. Soares PR, Hueb WA, Lemos PA, Lopes N, Martinez EE, Cesar LA, Oliveira SA, Ramires JA. Coronary revascularization (surgical or percutaneous) decreases mortality after the first year in diabetic subjects but not in nondiabetic subjects with multivessel disease: an analysis from the Medicine, Angioplasty, or Surgery Study (MASS II). Circulation. 2006;114(1 Suppl):1420-4

16. Benny Tu, Rich Ben, Christopher Labos CM, Brophy JM. Coronary revascularization in diabetic patients: a systematic review and Bayesian network meta-analysis. Ann Intern Med. 2014;161(10):724-32.

17. Verma S, Farkouh ME, Yanagawa B, et al. Comparison of coronary artery bypass surgery and percutaneous coronary intervention in patients with diabetes: a meta-analysis of randomized controlled trials. Lancet Diabetes Endocrinol. 2013;1:317-28.

18. Smit Y, Vlayen J, Koppenaal H, Eefting F, Kappetein AP, Mariani MA. Percutaneous coronary invervention versus coronary artery bypass grafting: a meta-analysis. J Thorac Cardiovasc Surg. 2015; 149(3):831-8.e1-13.

19. Marso SP, McGuire DK. Coronary revascularization strategies in patients with diabetes and multivessel coronary artery disease: has the final chapter been written? J Am Coll Cardiol. 2014;64(12):1198-201.

20. Wit MA, de Mulder M, Jansen EK, Umans VA. Diabetes mellitus and its impact on long-term outcomes after coronary artery bypass graft surgery. Acta Diabetol. 2013;50:123-8.

21. Lee $B$ J, Herbison P, Wong CK. Is the advantage of coronary bypass graft surgery over percutaneous coronary intervention in diabetic patientswith severe multivessel disease influenced by the status of insulin requirement? J Geriatr Cardiol. 2014;11(1):83-9.

22. Liu XJ, Wan ZF, Zhao N, Zhang YP, Mi L, Wang XH, Zhou D, Wu Y, Yuan ZY. Adjustment of the GRACE score by HemoglobinA1c enables a more accurate prediction of long-term majoradverse cardiac events in acute coronary syndrome without diabetes undergoing percutaneous coronary intervention. Cardiovasc Diabetol. 2015;14:110

23. Lopez-de-Andres A, Jimenez-García R, Hernandez-Barrera V, PerezFarinos N, de Miguel-Yanes JM, Mendez-Bailon M, Jimenez-Trujillo I, de Miguel AG, Pino CG, Carrasco-Garrido P. National trends in utilization and outcomes of coronary revascularization procedures among people with and without type 2 diabetes in Spain (2001-2011). Cardiovasc Diabetol. 2014;13:3.

24. Schuette C, Steffens D, Witkowski M, Stellbaum C, Bobbert P, Schultheiss $H P$, Rauch $U$. The effect of clopidogrel on platelet activity in patients with and without type-2 diabetes mellitus: a comparative study. Cardiovasc Diabetol. 2015:14:15.

25. Gargiulo G, Tamburino C, Capodanno D. Five-year outcomes of percutaneous coronary intervention versus coronary artery bypass graft surgery inpatients with left main coronary artery disease: An updated meta-analysis of randomized trials and adjusted observational studies. Int J Cardiol. 2015;195:79-81.

26. Hällberg V, Palomäki A, Lahtela J, Voutilainen S, Tarkka M. Kataja M; Study Group (W-CABG). Associations of metabolic syndrome and diabetes mellitus with 16-year survival after CABG. Cardiovasc Diabetol. 2014;13:25.

27. Naito R, Miyauchi K, Konishi H, Tsuboi S, Ogita M, Dohi T, Kajimoto K, Kasai T, Tamura H, Okazaki S, Isoda K, Yamamoto T, Amano A, Daida H. Comparing mortality between coronary artery bypass grafting and percutaneous coronary intervention withdrug-eluting stents in elderly with diabetes and multivessel coronary disease. Heart Vessels. 2015.

28. Bravata DM, Gienger AL, McDonald KM, et al. Systematic review: the comparative effectiveness of percutaneous coronary interventions and coronary artery bypass graft surgery. Ann Intern Med. 2007;147:703-16.

29. Daemen J, Boersma E, Flather $M$, et al. Long-term safety and efficacy of percutaneous coronary intervention with stenting and coronary artery bypass surgery for multivessel coronary artery disease: a meta-analysis with 5-year patient-level data from the ARTS, ERACI-II, MASS-II, and SoS trials. Circulation. 2008;118:1146-54.

30. Palmerini T, Biondi-Zoccai G, Reggiani LB, et al. Risk of stroke with coronary artery bypass graft surgery compared with percutaneous coronary intervention. J Am Coll Cardiol. 2012;60:798-805.

31. Tenenbaum A, Fisman EZ. Optimal revascularization in diabetes after the FREEDOM trial: were the controversies finally settled? Cardiol J. 2013;20(4):331-6.

\section{Submit your next manuscript to BioMed Central and we will help you at every step:}

- We accept pre-submission inquiries

- Our selector tool helps you to find the most relevant journal

- We provide round the clock customer support

- Convenient online submission

- Thorough peer review

- Inclusion in PubMed and all major indexing services

- Maximum visibility for your research

Submit your manuscript at www.biomedcentral.com/submit
Ciomed Central 